\title{
论文
}

\section{多重散射效应对部分相干源两粒子关联函数的 影响}

\author{
李淑侠，叶红安"，谢芳，宋秀丹* \\ 黑龙江大学电子工程学院, 哈尔滨 150080 \\ *联系人, 叶红安, Email: honganye@163.com; 宋秀丹, E-mail: songxiudan11@126.com \\ 收稿日期: 2014-12-11; 接受日期: 2015-01-21; 网络出版日期: 2015-01-30 \\ 国家自然科学基金资助项目(批准号: 11147016 和 11347139)
}

\begin{abstract}
摘要处理 HBT 多重散射的通常方法是经典二次碰撞的随机级联, 但是全同两粒子干涉实际是量子 效应，应该采用量子力学方法处理. 本文基于 Glauber 散射理论模型，利用量子路径积分方法，通过多 重散射对部分相干源发射函数作用一个吸收因子，来分析多重散射效应对部分相干源两粒子关联函数的 影响. 结果表明, 多重散射作用使关联函数的相角不为零从而引起粒子发射源的不对称性, 多重散射效 应使粒子很难从源内发出，粒子趋于表面发射，发射时间变短，所以多重散射作用使粒子发射源半径 减小.
\end{abstract}

关键词多重散射, 两粒子关联, 蒙特卡罗模拟计算

PACS: $25.75 .-\mathrm{q}, 25.75 . \mathrm{Nq}, 25.75 . \mathrm{Gz}$

doi: 10.1360/SSPMA2014-00477

\section{Glauber 理论在 $\pi \pi$ 散射中应用}

高能核-核碰撞必须要在重子层次上进行, 碰撞 后产生的高能类重子体可近似看成是沿入射方向继 续碰撞的入射体 ${ }^{[1-3]}$. 基于重子-重子碰撞后散射截面 值相同假设的 Glauber 模型能提供对碰撞核几何形状 的定量描述，可以近似的计算排对排的碰撞. 在高能 重离子碰撞中产生 $\pi$ 介子的多重数较多, 我们主要探 测和研究 $\pi$ 介子的产生与传播 ${ }^{[4,5]}$, 在 $\pi$ 介子从产生到 冻出的过程中会和媒介粒子(主要是 $\pi$ 介子)发生多重 散射相互作用, 当 $\pi$ 介子能量在 $300-1200 \mathrm{MeV}$ 范围
内时, Glauber 理论在研究介质粒子相互作用时得到 了很好的应用 ${ }^{[6-9]}$.

根据 Glauber 理论 ${ }^{[10,11]}$, 在描述碰撞的过程中我 们首先要确定靶粒子的初态 $k$ 和末态 $k^{\prime}$, 可以将碰撞 的散射振幅写成

$$
\begin{aligned}
& F_{\mathrm{fi} i}\left(k^{\prime}, k\right) \\
& =-\frac{2 m}{4 \pi \hbar^{2}} \int \mathrm{e}^{-\mathrm{i} k \cdot r} u_{\mathrm{f}}^{*}(q) V(r-q) \Psi_{\mathrm{k}, \mathrm{i}}(r, q) \mathrm{d} r \mathrm{~d} q .
\end{aligned}
$$

式中 $V(r-q)$ 是入射粒子与靶粒子相互作用的势场, $\Psi_{\mathrm{k}, \mathrm{i}}(r, q)$ 是入射粒子的波函数, $u_{\mathrm{f}}$ 是入射粒子的末

引用格式: 李淑侠, 叶红安, 谢芳, 等. 多重散射效应对部分相干源两粒子关联函数的影响. 中国科学: 物理学 力学 天文学, 2015, 45: 041002 Li S X, Ye H A, Xie F, et al. Effects of rescattering on two-particle correlation function of partially coherent sources (in Chinese). Sci Sin-Phys Mech Astron, 2015, 45: 041002, doi: 10.1360/SSPMA2014-00477 
态, $m$ 是入射粒子的质量, $r$ 是入射粒子的径向坐标, $q$ 是靶粒子坐标. 如果入射粒子能量较高远大于势 场 $V$, 入射粒子散射后的散射角较小, 带入入射粒子 的波函数, 并设 $s=q-k(k \cdot q), k^{\prime}-k=p$, 则(1)式可 以简写为

$$
F(p)=\frac{k}{2 \pi \mathrm{i}} \int \mathrm{e}^{-\mathrm{i} p \cdot b} u_{\mathrm{f}}^{*}(q)\left[\mathrm{e}^{\mathrm{i} \chi(b-s)}-1\right] u_{\mathrm{i}}(q) \mathrm{d} b \mathrm{~d} q .
$$

式中 $\chi$ 是势场造成的波函数的相移函数, 利用光学 原理对整个相空间积分, 可得到靶粒子碰撞后的散 射截面 ${ }^{[12]}$ :

$$
\sigma_{\mathrm{fi}}=\int_{\Omega_{\mathrm{f}}} \frac{k^{\prime}}{k}\left|F_{\mathrm{fi}}\left(k^{\prime}-k\right)\right|^{2} \mathrm{~d} \Omega_{k^{\prime}}
$$

$\sigma_{\mathrm{fi}}$ 就是散射截面, 单位是 $\mathrm{mb}, \Omega$ 称为立体角, 单位 是 sr. 靶粒子碰撞后的总散射截面就是对靶粒子的所 有可能末态求和, 利用 $\left|k^{\prime}\right|=k$ 和 $\mathrm{d} \Omega_{k^{\prime}} \rightarrow \mathrm{d}^{2} k / k^{2}$ 加上 完备性关系 $\sum_{\mathrm{f}} u_{\mathrm{f}}\left(q^{\prime}\right) u_{\mathrm{f}}^{*}(q)=\delta\left(q^{\prime}-q\right)$, 得到总散射截 面为 ${ }^{[9,12,13]}$

$$
\sigma_{\text {tot }}=\sum_{\mathrm{f}} \sigma_{\mathrm{fi}}=\int \mathrm{d} b \int\left|u_{\mathrm{i}}(q)\right|^{2}\left|\mathrm{e}^{\mathrm{i} \chi(b-s)}-1\right|^{2} \mathrm{~d} q .
$$

如果没有非弹性碰撞发生, 相移函数是与剖面函数 $\Gamma(b-s)$ 有关的实函数, 利用剖面函数总散射截面为

$$
\sigma_{\text {tot }}=2 \int \operatorname{Re} \Gamma(b-s) \mathrm{d} b,
$$

把 $\sigma_{\text {tot }}$ 乘以 $k / 4 \pi$ 与公式(2)给出的向前弹性散射振幅 进行比较, 可以把光学里的散射截面与散射振幅联 系起来, 并利用 $k^{\prime}-k=0$ 则总散射截面为

$$
\sigma_{\text {tot }}=\frac{4 \pi}{k} \operatorname{Im} F(0) \text {. }
$$

如果相移函数包含不为零的虚部 (如在某点上 $\operatorname{Im} V<0$, 表示粒子以一定的比率被吸收), 根据波函 数的幺正性, 公式(5)仍然成立.

当 $\pi$ 介子能量是从 300- $1200 \mathrm{MeV}$ 时可以把粒子 的轨迹近似地看成是直线, 设 $z$ 表示与被测粒子轨迹 平行的坐标, $x$ 表示与被测粒子轨迹垂直的坐标, $s$ 表 示与被测粒子轨迹垂直的媒介粒子坐标, $\theta$ 是阶梯 函数, 实函数 $\chi(b-s)$ 可以写成

$$
\chi(b-s)=\sum_{j=1}^{N} \chi\left(x_{f}-s_{j}\right) \theta\left(z_{f}-z_{j}\right) \theta\left(z_{j}-z\right) .
$$

粒子从源点产生到冻出的过程中与媒介粒子相互作 用后波函数的相移:

$$
\begin{aligned}
& \exp \left\{\mathrm{i} \varphi_{s}\left(x-x^{\prime}\right)\right\} \\
& =\exp \left\{\mathrm{i} \sum_{j=1}^{N} \chi\left(x_{\perp}^{\prime}-s_{j \perp}\right) \theta\left(x_{\|}^{\prime}-s_{j \|}\right) \theta\left(s_{j \|}-x_{\|}\right)\right\} .
\end{aligned}
$$

$j=1$ 到 $N$ 求和后, 从两体散射数据和横向碰撞参数 可以得到相移函数 $\chi(b)$. 这里的 $\chi\left(x_{\perp}^{\prime}-s_{j \perp}\right)$ 是被测 粒子和介质粒子在源内的点 $s_{j}$ 发生碰撞后的 Glauber 相移函数, $s_{j}$ 是关于 $x$ 的函数, 所以上式都表示成关 于 $x$ 的函数. 由(7)式可以看到相移函数包含实部和 虚部, 虚部表示被测粒子与媒介粒子多次碰撞后被 测粒子被吸收的程度. 设 $b$ 是描述与粒子入射方向 垂直平面内位置的量, 根据被测粒子与媒介粒子碰 撞的散射数据可以得到散射截面 ${ }^{[11]}$ :

$$
\sigma_{\text {tot }}=\int\left|u_{\mathrm{i}}(q)\right|^{2}\left|\mathrm{e}^{2 \mathrm{i} x\left(b-b_{j}\right)}-1\right|^{2} \mathrm{~d} q .
$$

假设被测粒子与媒介粒子的相互作用区域小于介质 的尺寸, 相移函数可以写成

$$
\exp \left\{2 \mathrm{i} \chi\left(b-b_{j}\right)\right\} \approx 1+2 \mathrm{i} \chi(b) .
$$

把(9)式带入 (8) 式并利用 $u_{i}(q)$ 的完备性关系可以 得到

$$
\chi\left(b-b_{j}\right)=\frac{\mathrm{i} \sigma_{\mathrm{tot}}}{2} \delta\left(b-b_{j}\right) .
$$

这里的 $\chi\left(b-b_{j}\right)$ 是复数的虚部, 与前面的 Glauber 理 论联系在一起, 它们都可以表示相移. 利用多重散射 效应对粒子谱分布的影响, 通过对所有媒介粒子的 坐标取平均后把多重散射效应加入到动量谱分布中 得到 ${ }^{[9]}$

$$
\begin{aligned}
& \mathrm{e}^{-2 \operatorname{Im} \bar{\varphi}_{s}(x)} \\
& =\int \mathrm{d} x_{2} \mathrm{~d} x_{3} \cdots \mathrm{d} x_{N} \mathrm{e}^{-2 \operatorname{Im} \varphi_{2}(x)} \rho_{\text {med }}\left(x_{2}, x_{3} \cdots x_{N}\right), \\
& \left\langle\mathrm{e}^{-2 \operatorname{Im} \varphi_{s}(x)}\right\rangle=\exp \left\{-\int_{x}^{x_{f}} n(x) \sigma_{\mathrm{tot}} \mathrm{d} l\right\} .
\end{aligned}
$$

由(12)式我们可以知道被测粒子与媒介粒子碰撞后 相当于对被测粒子的源密度函数产生了吸收, 其吸 收程度与吸收截面和媒介粒子的数密度积分有关, 吸收效应将限制被测粒子从粒子发射源的中心发 射, 粒子趋于表面发射, 即粒子发射具有不透明机 制 ${ }^{[14-16]}$, 因而将使 HBT 半径减小.

\section{2 经典流系综模型下的两粒子关联函数}

两粒子关联函数定义为测量到两个动量为 $k_{1}$ 和 
$k_{2}$ 的 $\pi$ 介子的几率和分别观察到 $k_{1}$ 和 $k_{2}$ 的几率之 比，即

$$
C\left(k_{1}, k_{2}\right)=\frac{P\left(k_{1}, k_{2}\right)}{P\left(k_{1}\right) P\left(k_{2}\right)} .
$$

Heinz 等人 ${ }^{[13]}$ 将发射函数 $S(x, k)$ 定义为

$$
S(x, k)=\int \mathrm{d}^{4} x^{\prime} \eta^{*}\left(x+\frac{1}{2} x^{\prime}, t\right) \eta\left(x-\frac{1}{2} x^{\prime}, t\right) \mathrm{e}^{\mathrm{i} k \cdot x^{\prime}},
$$

由发射函数表示的关联函数形式为 ${ }^{[17]}$

$$
\begin{aligned}
& C\left(k_{1}, k_{2}\right) \\
& =1+\frac{\int \mathrm{d}^{4} x \mathrm{~d}^{4} x^{\prime} S(x, K) S\left(x^{\prime}, K\right) \exp [-\mathrm{i} q \cdot x]}{\int \mathrm{d}^{4} x \mathrm{~d}^{4} x^{\prime} S\left(x, k_{1}\right) S\left(x^{\prime}, k_{2}\right)} .
\end{aligned}
$$

其中 $K=\frac{k_{1}+k_{2}}{2}, q=k_{1}-k_{2}$. 粒子发射源的发射函 数 $S(x, k)$ 表示在时空点 $x$ 发射动量为 $k$ 的几率, 它已 不再具有经典意义下的解释, 从(14)式中可以看出关 联函数这一可观测量能确定粒子发射源的分布函数, 这种方式表达的关联函数公式在物理图像上直观, 便于我们分析和模拟计算.

把发射函数的时空表达式转换成动量时空表达 式, 并引入部分相干源的概念, 可以得到部分相干源 的发射函数形式为 ${ }^{[17]}$

$$
\begin{aligned}
S(x, K) & =\int \frac{\mathrm{d}^{4} y}{2(2 \pi)^{3}} \mathrm{e}^{-\mathrm{i} K \cdot y}\left\langle J^{*}\left(x+\frac{y}{2}\right) J\left(x-\frac{y}{2}\right)\right\rangle \\
& =S_{\text {coh }}(x, K)+S_{\text {cha }}(x, K) .
\end{aligned}
$$

式中 $S_{\mathrm{coh}}(x, K)$ 为相干部分的发射函数, $S_{\mathrm{cha}}(x, K)$ 为 混沌部分的发射函数, 即

$$
\begin{aligned}
& S_{\text {coh }}(x, K) \\
& =\int \frac{\mathrm{d}^{4} y}{2(2 \pi)^{3}} \mathrm{e}^{-\mathrm{i} K \cdot y}\left\langle J_{\text {coh }}^{*}\left(x+\frac{y}{2}\right) J_{\text {coh }}\left(x-\frac{y}{2}\right)\right\rangle, \\
& S_{\text {cha }}(x, K) \\
& =\int \frac{\mathrm{d}^{4} y}{2(2 \pi)^{3}} \mathrm{e}^{-\mathrm{i} K \cdot y}\left\langle J_{\text {cha }}^{*}\left(x+\frac{y}{2}\right) J_{\text {cha }}\left(x-\frac{y}{2}\right)\right\rangle .
\end{aligned}
$$

根据源相干部分和混沌部分的物理意义, 可以将源 Wigner 密度函数写成是相干部分和混沌部分之和 ${ }^{[17]}$ :

$$
\begin{aligned}
\rho_{i j} & =\int \mathrm{d}^{4} x S(x, K) \mathrm{e}^{\mathrm{i} q_{i j} x}=\rho_{i j}^{\text {cha }}+\rho_{i j}^{\text {coh }} \\
& \equiv F_{i j} \mathrm{e}^{\mathrm{i} \varphi_{i j}}+f_{i j} \mathrm{e}^{\mathrm{i} \varphi_{i j}} .
\end{aligned}
$$

并且

$$
\begin{aligned}
& F_{i j} \mathrm{e}^{\mathrm{i} \Phi_{i j}}=\int \mathrm{d} x S_{\text {cha }}\left(x, K_{i j}\right) \mathrm{e}^{\mathrm{i} q_{i j} \cdot x}, \\
& f_{i j} \mathrm{e}^{\mathrm{i} \varphi_{i j}}=\int \mathrm{d} x S_{\text {coh }}\left(x, K_{i j}\right) \mathrm{e}^{\mathrm{i} q_{i j} \cdot x} .
\end{aligned}
$$

引入相干源的相角 ${ }^{[17]}, \Phi=\Phi_{12}-\varphi_{1}+\varphi_{2}$, 并利用平均 值的概念，可以得到部分相干源发射函数为

$$
\begin{aligned}
C\left(k_{1}, k_{2}\right)= & 1+2 \sqrt{p_{1}\left(1-p_{1}\right) p_{2}\left(1-p_{2}\right)} T_{12} \cos \Phi \\
& +p_{1} p_{2} T_{12}^{2} .
\end{aligned}
$$

\section{3 多重散射效应对部分相干源关联函数的 影响}

在核-核碰撞中当一个入射核子受到碰撞后，由 于核物质的高密度而使生成的高能类重子体发生多 重散射, 在 Glauber 理论中, 正确处理有无多重散射 的方法是将无散射对应于一系列多重散射的前 $N-1$ 次 ${ }^{[9,12]}$. 被测粒子从产生到经过媒介粒子的过程中, 与媒介粒子的浓厚没有什么必然的联系，媒介粒子 附近的浓厚和远处媒介粒子的稀薄一样, 因为在计 算散射截面时是对整个粒子发射源内的所有介质粒 子积分所得.

由前面讨论知道多重散射是通过作用一个吸收 因子影响源粒子密度函数, 我们将多重散射对部分 相干源关联函数的影响写成对发射函数作用一个吸 收因子, 所以

$$
g^{\prime}\left(r^{\prime}, k^{\prime}\right)=g(r, k)\left|\mathrm{e}^{\mathrm{i} \varphi_{s}\left(r-r^{\prime}\right)}\right|^{2}=g(r, k) \mathrm{e}^{-2 \operatorname{Im} \varphi_{s}} .
$$

其中相移 $\varphi_{s}\left(r \rightarrow r^{\prime}\right)=\chi_{s}\left(r \rightarrow r^{\prime}\right), \quad \operatorname{Im}$ 表示取复数的 虚部, 由前面讨论可以知道 $\chi_{s}\left(r \rightarrow r^{\prime}\right)$ 是因多重散射

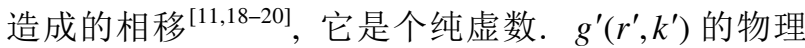
意义就是考虑被测粒子与媒介粒子间的多重散射后 而得到的发射函数. 考虑了多重散射效应后的 Wigner 发射函数为

$$
S(x, k)=\int g(r, k) \mathrm{e}^{\mathrm{i} q \cdot x} \mathrm{e}^{-2 \operatorname{Im} \varphi_{s}} \mathrm{~d} r .
$$

将公式(23)代入公式(19)并利用平均值的概念可以得 到

$$
F_{i j} \mathrm{e}^{\mathrm{i} \phi_{i j}}=\int \mathrm{d} x S_{\text {cha }}\left(x, K_{i j}\right)\left\langle\mathrm{e}^{\mathrm{i} q_{i j} \cdot x} \mathrm{e}^{-2 \operatorname{Im} \varphi_{s}}\right\rangle .
$$

由上式可以看出, 多重散射对发射函数作用的吸收 因子, 可以影响部分相干源关联函数的相角. 这个吸 收因子在前面已经讨论过了，它与媒介粒子发生碰 撞后的散射截面和源的粒子数密度有关, 但是 $\mathrm{e}$ 指数 
上的 $-2 \operatorname{Im} \varphi_{s}$ 是个虚部, 所以可以将其写成如下形式:

$$
\left\langle\mathrm{e}^{\mathrm{i} q \cdot x} \mathrm{e}^{-2 \operatorname{lm} \varphi_{s}}\right\rangle=\left\langle\mathrm{e}^{\mathrm{i} q \cdot x+\left(-2 \operatorname{lm} \varphi_{s}\right)}\right\rangle \text {. }
$$

根据平均值的概念, 令 $-2 \operatorname{Im} \varphi_{s}=A$, 因为 $A$ 表示的 是对被测粒子与粒子发射源内所有媒介粒子相互作 用后的坐标求平均的结果, 由散射截面公式可以知 道它表示的是散射截面与粒子数密度的乘积的积分, 是个微小的量. 所以根据平均值的定义可以将(25)式 按照 $\mathrm{e}$ 指数展开成如下形式:

$$
\begin{aligned}
\left\langle\mathrm{e}^{\mathrm{i} q \cdot x+\left(-2 \mathrm{Im} \varphi_{s}\right)}\right\rangle=\left\langle\mathrm{e}^{\mathrm{i} q \cdot x+A}\right\rangle & \\
= & \left\langle 1+(\mathrm{i} q \cdot x+A)+\frac{(\mathrm{i} q \cdot x+A)^{2}}{2}+\frac{(\mathrm{i} q \cdot x+A)^{3}}{6}\right\rangle \\
= & \left\langle 1+A+\frac{A^{2}}{2}+\frac{1}{6} A^{3}-\frac{1}{2}(q \cdot x)^{2}-\frac{1}{2} A(q \cdot x)^{2}\right\rangle \\
& +\mathrm{i}\left\langle(q \cdot x)+A(q \cdot x)-\frac{1}{6}(q \cdot x)^{3}+\frac{1}{2} A^{2}(q \cdot x)\right\rangle .
\end{aligned}
$$

根据部分相干源相角的定义式, 得到多重散射作用 的部分相干源相角的表达式为

$$
\begin{aligned}
\tan \Phi^{\prime}= & \frac{\left\langle(q \cdot x)+A(q \cdot x)-\frac{1}{6}(q \cdot x)^{3}+\frac{1}{2} A^{2}(q \cdot x)\right\rangle}{\left\langle 1+A+\frac{1}{2} A^{2}+\frac{1}{6} A^{3}-\frac{1}{2}(q \cdot x)^{2}-\frac{1}{2} A(q \cdot x)^{2}\right\rangle} \\
= & {\left[1+2 A+2 A^{2}+\frac{7}{6} A^{3}+\frac{5}{12} A^{4}+\frac{1}{12} A^{5}\right]\langle q \cdot x\rangle } \\
& +\left[\frac{1}{2}-\frac{1}{4} A^{2}-\frac{1}{4} A^{3}\right]\left\langle(q \cdot x)^{2}\right\rangle\langle q \cdot x\rangle \\
& -\frac{1}{6}\left(1+A+\frac{1}{2} A^{2}+\frac{1}{6} A^{3}\right)\left\langle(q \cdot x)^{3}\right\rangle .
\end{aligned}
$$

设 $\tan \Phi^{\prime}=u$, 根据 $\Phi^{\prime}=u-\frac{u^{3}}{3}$

$$
\begin{aligned}
\Phi^{\prime}= & {\left[1+2 A+2 A^{2}\right]\langle q \cdot x\rangle } \\
& +\frac{1}{6}\left[1+A+\frac{A^{2}}{2}\right]\left\langle(q \cdot \tilde{x})^{3}\right\rangle+\mathrm{O}(q \cdot x)^{5}, \\
F_{i j} \mathrm{e}^{\mathrm{i} \Phi}= & P_{1}^{\mathrm{ch}}\left(K_{i j}\right)\left[\left(1+A+\frac{1}{2} A^{2}\right)\right. \\
& \left.-\frac{1}{6}\left(1+A+\frac{1}{2} A^{2}\right)\left\langle(q \cdot \tilde{x})^{3}\right\rangle_{i j}+\mathrm{O}\left(q_{i j}\right)^{4}\right] .
\end{aligned}
$$

其中 $P_{1}^{\mathrm{ch}}\left(K_{i j}\right)$ 的含义见文献[17], $\tilde{x}=x-\langle x\rangle_{i j}$.

从上面相角表达式(28)中可以看出, 考虑了多重 散射效应后的相角多乘了一个与相移有关的量. 相
角 $\Phi$ 与粒子发射源的不对称性密切相关, 所以多重 散射效应也是引起发射函数不对称的物理效应之一, 由(21)和(28)式还可以看出多重散射吸收因子通过相 角也会影响部分相干源两粒子关联函数，从而影响 粒子发射源的时空结构.

\section{4 多重散射效应部分相干源关联函数的蒙 特卡罗模拟计算}

通过蒙特卡罗方法获得关联 $\pi$ 介子对可以这样 实现的 ${ }^{[21]}$ : HBT 关联函数依赖粒子发射函数, 发射函 数是对物理图像的一种假定，即发射函数是根据物 理事实构造出来的，由已经得到的多重散射作用的 发射函数形式，选用高斯型粒子发射源获得关联 $\pi$ 介 子对. $\pi$ 介子的动量分布是根据火球模型引入，如果 高能核-核碰撞过程中产生的粒子达到了完全热平衡 条件, $\pi$ 介子可以看作理想的玻色气体，这样就可以 用玻尔兹曼分布来描述 $\pi$ 介子的动量分布. 利用流 体动力学模型的初始条件和源的演化过程求解每一 次碰撞后的粒子的新的坐标和动量, 在相对动量差 很小范围内 $\pi$ 介子的关联对数占所有关联对数的很 大几率, 所以在理论上可以将满足动量差很小的 $\pi$ 介子对作为我们研究的对象, 利用小相对动量差区 域的关联函数只和源的半径平均值有关, 我们就可 以计算源的半径. 对多重散射效应部分相干源关联 函数的模拟计算同样采用文献[17]的计算方法，即

$$
\begin{gathered}
F_{12}^{2} \rightarrow \sum_{i j \in \operatorname{bin}(K, \varepsilon)} \cos \left(q \cdot\left(x_{i}-x_{j}\right)\right), \\
2 F_{12} f_{12} \cos \Phi \rightarrow \sum_{i j \in \operatorname{bin}(K, \varepsilon)} \cos \left(q \cdot\left(x_{i}+x_{j}^{\prime}\right)\right) .
\end{gathered}
$$

式中 $x_{i}$ 为混沌源发射粒子的坐标, $x_{j}$ 为相干源发射 粒子的坐标, 选取关联粒子对动量必须在小动量差 范围内以保证发射函数中的动量为近似在壳上, 关 联函数就可以通过对它们累加求和得到. 多重散射 是在粒子发射源的演化过程中进行的核子间的相互 作用, 为了得到具体的结果, 本文用流体动力学模型 的初始条件、状态方程和冻结的时间来求解源演化不 同时期的粒子坐标和动量. 流体动力学模型不能描 述源的碰撞初期和碰撞末期, 所以这里用 $T^{\text {th }}$ 和 $T^{\mathrm{ch}}$ 分别表示粒子发射源热冻结时的温度和粒子化学冻 出时的温度. 粒子发射源的演化在流体动力学模型 下可以由状态方程确定，在给定了初始条件和冻结 
条件后我们就可以计算粒子从产生到冻出过程中的 坐标和动量. 本文在 $\mathrm{AGS}$ 能量下, 设置初始条件为: 初始源半径 $R=6.0 \mathrm{fm}$, 初始温度 $T_{0}=155 \mathrm{MeV}$, 初 始重子化学势 $\mu_{B 0}=540 \mathrm{MeV}$, 对应的初始能量密度 $\varepsilon_{0}=0.56 \mathrm{GeV} / \mathrm{fm}^{3}$, 化学冻出温度 $T^{\mathrm{ch}}=124 \mathrm{MeV}$, 该 温度下的重子化学势为 $\mu_{B}^{\mathrm{ch}}=565 \mathrm{MeV}$, 热冻出温度 $T^{\text {th }}=70 \mathrm{MeV}$, 这些值是根据 $\mathrm{AGS}$ 上的实验设定的, 该蒙特卡罗模拟计算的程序框图如图 1 所示.

本文模拟计算了多重散射效应对部分相干源两 粒子关联函数和关联函数相角的影响, 为了计算简 便, 忽略了粒子发射源的相干部分对相角 $\Phi$ 的影响, 利用对粒子发射源内所有媒介粒子的吸收截面与粒 子数密度的乘积累加求和, 可以计算出散射截面及 多重散射的吸收因子. 多重散射效应对部分相干源 关联函数相角 $\Phi$ 影响的模拟结果如图 2, 图中纵坐标 为相角 $\Phi$ 的余弦 $\cos \Phi$, 横坐标为两粒子的相对动量 $q$, 图 2 中方块符号表示根据公式(28)计算的相角理 论值, 星状符号表示根据公式(21)，(30)，(31)得到的 相角蒙特卡罗模拟计算值, 实线是对相角蒙特卡罗 模拟计算值的拟合, (a)图表示静态高斯源, (b)-(d)分 别表示不透明、多重散射、不透明和多重散射效应的 高斯源. 对于静态高斯型的粒子发射源发射函数是 对称的, 所以 (a)图相角为零, $\cos \Phi=1$. 但核-核碰撞 区域附近能量密度很高, 源内部粒子容易发生多重 散射, 粒子很难在源的内部冻出, 即粒子趋于源的表 面发射形成不透明效应, 所以发射函数是不对称, 相 角不为零, $\cos \Phi \neq 1$, 如图(b)-(d)所表示.

图 3 给出了多重散射效应对不同混沌程度部分 相干源两粒子关联函数的影响, 其中实线表示静态 高斯源的关联函数，(a)-(c)和(d)-(f)中的虚线分别表 示不透明、多重散射、不透明和多重散射效应高斯源 的关联函数, (a)-(c)表示部分相干源关联函数的混沌 程度 $p=0.3$, (d)-(f) 表示的混沌程度 $p=0.7$. 因为两粒 子关联函数与粒子发射源的空间大小 $R$ 有关,

$$
R^{2}=\left.\frac{\mathrm{d}^{2} C(q)}{\mathrm{d} q^{2}}\right|_{q=0},
$$

所以计算结果表明, 不透明、多重散射效应使粒子发 射源的半径减小. 为了从两粒子关联函数中获得粒 子发射源大小的具体信息, 本文对部分相干源关联 函数进行高斯形式的参数化拟合, 拟合函数 $C_{2}(q)=$

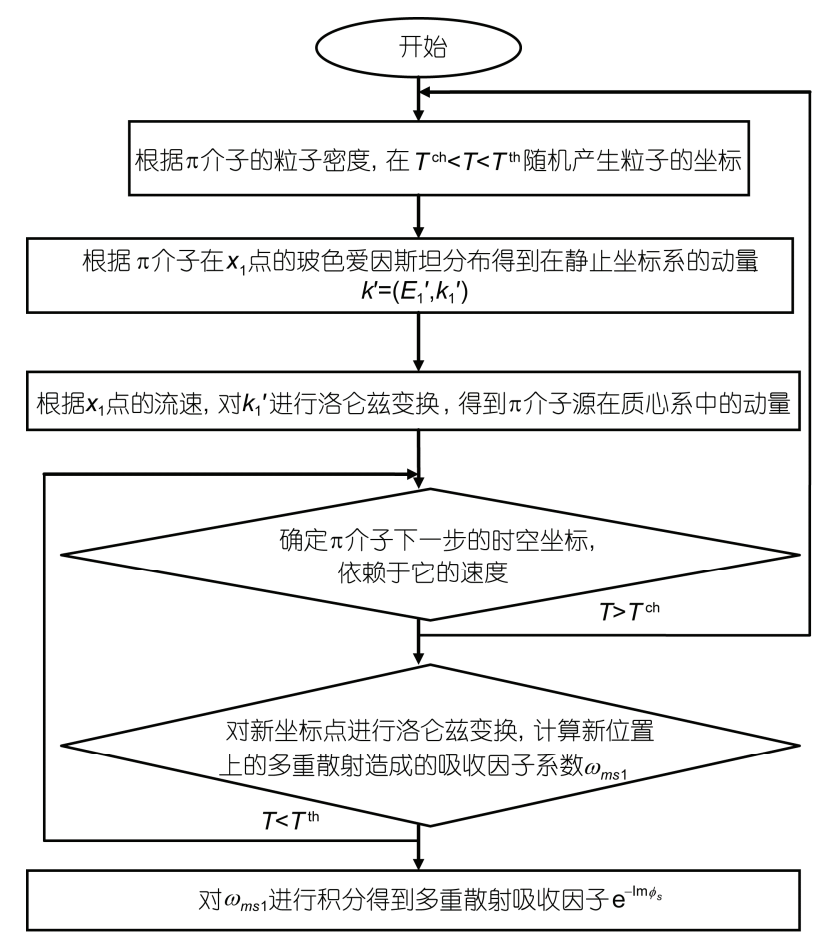

图 1 用蒙特卡罗方法计算多重散射影响因子程序框图

Figure 1 The program flow chart of calculating rescattering factor by Monte-Carlo simulating.

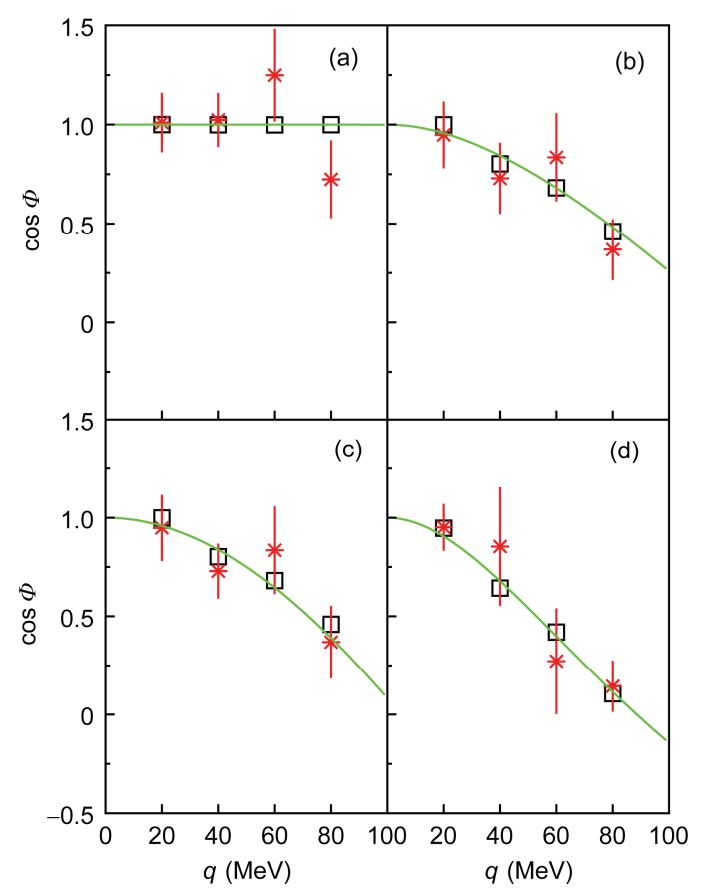

图 2 (网络版彩图)不透明和多重散射效应对部分相干源关 联函数相角的影响

Figure 2 (Color online) The effect of opacity and rescattering on phase for partially coherent sources. 


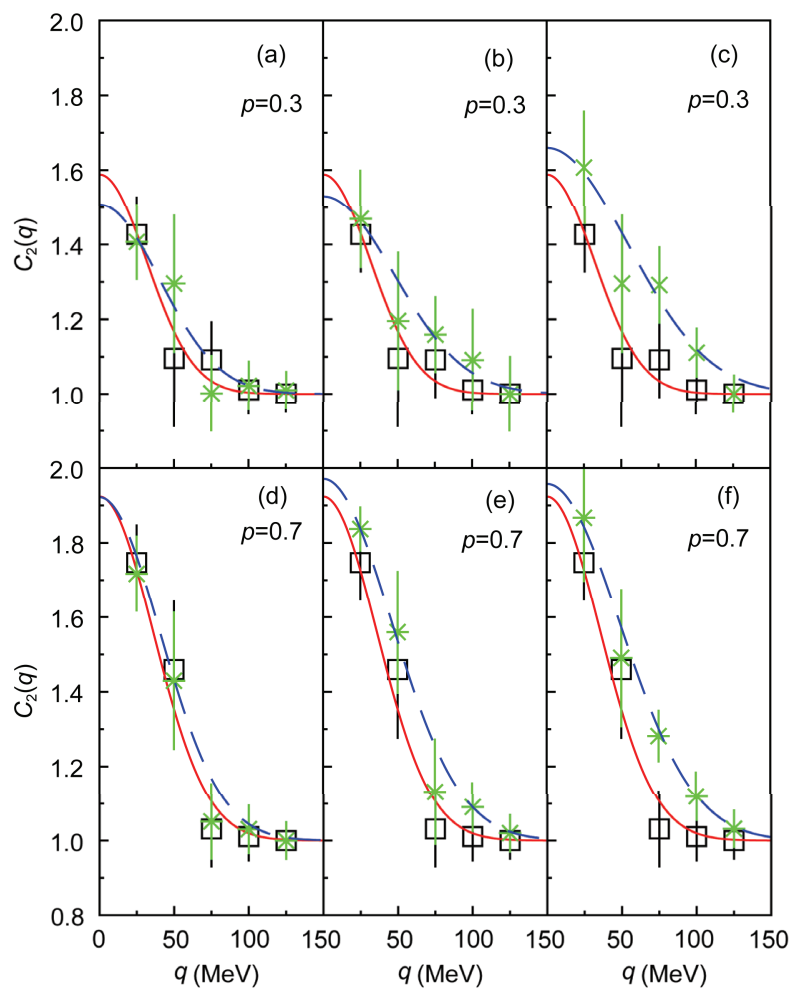

图 3 (网络版彩图)不透明和多重散射效应对部分相干源两 粒子关联函数的影响

Figure 3 (Color online) The effects of opacity and rescattering on two-particle correlation function for partially coherent sources.

$1+\lambda \mathrm{e}^{-q^{2} R^{2}}$, 拟合结果如表 1 , 结果表明多重散射效应 使粒子发射源的半径减小, 即粒子趋于表面发射. 在 高能核-核碰撞中，根据 Glauber 理论粒子在源的内部 要完成多次碰撞, 碰撞效果是通过多重散射使粒子 之间不断地交换动量和能量, 膨胀演化源趋于混沌. 根据(23)-(25)式的讨论, 被测粒子和源内粒子的多重 散射作用是对发射函数施加了一个吸收因子，如果 在源的内部粒子不断进行多重散射, 粒子很难从源 的内部发出, 所以多重散射效应限制粒子在中心区 域发射，粒子趋于表面发射，发射时间变短，所以粒
表 1 两粒子关联函数的高斯型参数化拟合半径, 源的初 始半径 $R=6 \mathbf{f m}$

Table 1 The Gaussian fitting radius of two-particle correlation function for initial $R=6 \mathrm{fm}$

\begin{tabular}{|c|c|c|c|}
\hline$P_{\text {chaoticparameter }}$ & $R$ 不透明 & $R$ 多重政射 & $R$ 不透明十多重散射 \\
\hline 0.3 & 5.781 & 5.378 & 5.025 \\
\hline 0.7 & 5.794 & 5.694 & 5.086 \\
\hline
\end{tabular}

子发射源的半径减小.

本文是在流体动力学模型下求解被测粒子与媒 介粒子作用后的动量和坐标, 再通过洛仑兹变换计 算被测粒子在质心系下新的动量和坐标, 并对每一 次碰撞的散射截面与粒子数密度的乘积累加求和后 得到吸收因子，没有受到多重散射作用的两粒子关 联函数也是在流体动力学模型下计算的.

\section{5 结论}

本文利用 Glauber 散射理论模型, 研究量子力 学机制下多重散射效应对部分相干源两粒子关联函 数的影响. 在粒子发射源的演化后期, 被测粒子在传 播过程中要受到媒介粒子的多重散射作用, 多重散 射对部分相干源发射函数作用一个吸收因子，从而 影响末态探测到粒子的几率, 最终影响干涉学的 HBT 结果. 对于部分相干源的两粒子关联函数, 多 重散射作用使相角多乘了一个与相移有关的量, 导 致相角不为零, 从而引起粒子发射源的不对称性. 在 源内部多重散射效应使粒子之间不断地交换动量和 能量, 膨胀演化源趋于混沌, 多重散射限制粒子从源 的内部发出，粒子趋于表面发射，发射时间变短，所 以多重散射作用下的粒子发射源半径减小。利用蒙 特卡罗模拟方法计算了不透明和多重散射效应对部 分相干源的两粒子关联函数和相角的影响，计算结 果表明多重散射效应限制粒子在中心区域发射，考 虑多重散射效应的粒子发射源演化过程更加符合真 实情况.

\section{参考文献}

1 Habich M, Nagle J L, Romatschke $\mathrm{P}$, et al. Particle spectra and HBT radii for simulated central nuclear collisions of $\mathrm{C}+\mathrm{C}, \mathrm{Al}+\mathrm{Al}, \mathrm{Cu}+\mathrm{Cu}$, $\mathrm{Au}+\mathrm{Au}$, and $\mathrm{Pb}+\mathrm{Pb}$ from $\mathrm{Sqrt}(\mathrm{s})=62.4-2760 \mathrm{GeV}$. arXiv: 1409.0040

2 PHENIX Collaboration. Detailed HBT measurements with respect to the event plane and collision energy in Au+Au collisions at PHENIX. Nucl Phys A, 2013, 904-905: 439c-442c 
3 Bialas A, Florkowski W, Zalewski K, et al. Bose-Einstein correlations and thermal cluster formation in high-energy collisions. Acta Phys Polon B, 2014, 45: 1883

4 Csernai L P, Velle S, Wang D J, et al. Differential HBT applied to relativistic fluid dynamics. Phys Rev C, 2014, 89: 034916

5 Abelev B, Adam J, Adamová D, et al. ALICE Collaboration. Two- and three-pion quantum statistics correlations in $\mathrm{Pb}-\mathrm{Pb}$ collisions at $\sqrt{S_{N N}}=2.76 \mathrm{TeV}$ at the LHC. Phys Rev C, 2014, 89: 024911

6 Wong C Y, Zhang W N. Chaoticity parameter $\lambda$ in Hanbury-Brown-Twiss interferometry. Phys Rev C, 2007, 76: 034905

7 Wong C Y. Intensity interferometry for a chaotic source with a collective flow and multiple scattering. J Phys G-Nucl Partical Phys, 2003 , 29: 2151-2168

8 Wong C Y. Quantum treatment of the multiple scattering and collective flow in intensity interferometry. AIP Conf Proc, 2006, 828: $617-622$

9 Yu L L, Zhang W N, Wong C Y, et al. HBT interferometry with quantum transport of the interfering pair. Phys Rev C, 2008, 78: 014908

10 Pelaez J R, Yndurain F J. Pion-pion scattering amplitude. Phy Rev D, 2005, 71: 074016

11 Kapusta J, Li Y. Rescattering effects on HBT interferometry. J Phys G-Nucl Partical Phys, 2004, 30: S1069-S1072

12 Wong C Y. Signatures of quark-gluon plasma phase transition in high-energy nuclear collisions. Nucl Phys A, 2001, 681: 22-33

13 Heinz U, Kolb P F. Two RHIC puzzles: Early thermalzation and the HBT problem. arXiv: hep-ph/0204061

14 Molnar D, Gyulassy M. Differential freeze-out and pion interferometry at RHIC from covariant transport theory. Phys Rev Lett, 2004, 92: 052301

15 Andronic A, Braun-Munzinger P, Stachel J, et al. Hadron production in central nucleus-nucleus collisions at chemical freeze-out. Nucl Phys A, 2006, 772: 167-199

16 Tomásik B. DRAGON: Monte Carlo generator of particle production from a fragmented fireball in ultrarelativistic nuclear collisions. Comput Phys Commun, 2009, 180: 1642-1653

17 Li S X, Sun P N, Xiao D H, et al. Effects of source opacity and expansion on two-pion Bose-Einstein correlations. Sci China-Phys Mech Astron, 2011, 54 (5): 909-915

18 Morita K, Muroya S, Nakamura H, et al. Source chaoticity in relativistic heavy-ion collisions at SPS and RHIC. Braz J Phys, 2007,37 : 705-707

19 Li Q F. Pion freeze-out through HBT correlation in RHIC from AGS/FAIR to RHIC energies. arXiv: 0708.2613

20 Yu L L, Ji R M T, Zhang W N, et al. Two-pion Interferometry in heavy ion collisions at HIRFL-CSR energy. Chin Phy C, 2008, 32: 897-902

21 Armesto N. Review of Monte Carlo methods for particle multiplicity evaluation. J Phys Conf Ser, 2005, 5: 219-229 


\title{
Effects of rescattering on two-particle correlation function of partially coherent sources
}

\author{
LI ShuXia, YE HongAn*, XIE Fang \& SONG XiuDan* \\ College of Electronic Engineering, Heilongjiang University, Harbin 150080, China
}

The normal method handling the HBT rescattering effect is classical random cascade of secondary collision in high energy heavy ion collisions program whose goal is to seek the quark-gluon plasma. Interferometry, also called Hanbury-Brown Twiss (HBT) effects, is an important tool to study the space-time feature of particle-emitting source, and is an effective method to examine the quantum chromodynamics phase transition. As the HBT interference phenomenon of two identical particles is quantum mechanical in nature, and HBT results are affected by the particleemitting source collective expansion, excited-particle decays and rescattering of particles in the source, hence, these effects should be studied properly in quantum mechanics frame. In this thesis, based on Glauber's scattering model, we use the method of path integral to analysis the rescattering effect on two-particle correlation functions of the partially coherent sources and give the formulas of two-particle correlation functions adding a absorbing factor due to rescattering effect by studying the quantum transport of the interfering identity particle pair, which include the rescattering effect. The two-particle correlation functions of the partially coherent sources contain a phase which is sensitive to the asymmetry of the source emission function and the rescattering effect shift the phase from zero and cause asymmetry of particle emission source. We investigate the influence of rescattering in high energy heavy ion collision on two-particle correlation functions for the partially coherent sources by Monte Carlo calculations. The result shows that the rescattering effect makes the phase not equal zero, causing asymmetry of particle emission source. The rescattering effect makes particles hard to emit from the center of sources, particles tend to emit from the surface of sources and the emitting time shorten. The Gaussian-formula fit results to the simulated two-particle correlation functions indicate that rescattering effect leads to a smaller interferometry radius due a shorter emitting time.

rescattering effect, two-particle correlation function, Monte-Carlo simulating method

PACS: $25.75 .-\mathrm{q}, 25.75 . \mathrm{Nq}, 25.75 . \mathrm{Gz}$

doi: $10.1360 /$ SSPMA2014-00477 